OPEN

\author{
SUBJECT AREAS:
}

ENGINEERING

MATHEMATICS AND

COMPUTING

Received

5 August 2014

Accepted

29 September 2014

Published

15 October 2014

Correspondence and requests for materials should be addressed to

J.L. (lujing@nju.edu.cn) or J.C. (jccheng@nju.

edu.cn)

* These authors contributed equally to this work.

\section{Delivering Sound Energy along an Arbitrary Convex Trajectory}

\author{
Sipei Zhao ${ }^{1,2 *}$, Yuxiang Hu ${ }^{1 *}$, Jing Lu' ${ }^{1}$ Xiaojun Qiu ${ }^{1,2}$, Jianchun Cheng ${ }^{1} \&$ lan Burnett ${ }^{2}$
}

\begin{abstract}
${ }^{1}$ Key Laboratory of Modern Acoustics, Department of Physics, Collaborative Innovation Centre for Advanced Microstructure, Nanjing University, Nanjing 210093, P. R. China, ${ }^{2}$ School of Electrical and Computer Engineering, RMIT University, Melbourne 3000, Australia.
\end{abstract}

Accelerating beams have attracted considerable research interest due to their peculiar properties and various applications. Although there have been numerous research on the generation and application of accelerating light beams, few results have been published on the generation of accelerating acoustic beams. Here we report on the experimental observation of accelerating acoustic beams along arbitrary convex trajectories. The desired trajectory is projected to the spatial phase profile on the boundary which is discretized and sampled spatially. The sound field distribution is formulated with the Green function and the integral equation method. Both the paraxial and the non-paraxial regimes are examined and observed in the experiments. The effect of obstacle scattering in the sound field is also investigated and the results demonstrate that the approach is robust against obstacle scattering. The realization of accelerating acoustic beams will have an impact on various applications where acoustic information and energy are required to be delivered along an arbitrary convex trajectory.

elf-accelerating beams have attracted considerable research interest in optics since the concept of the Airy beam was introduced from quantum mechanics ${ }^{1}$ into optics in $2007^{2,3}$. As an exact solution of the paraxial wave equation, the Airy beam propagates along parabolic trajectories without diffraction ${ }^{1,2}$. In the past few years, the Airy beam has been studied extensively and possible applications have been proposed and demonstrated, such as guiding microparticles ${ }^{4}$, producing curved plasma channels ${ }^{5}$ and routing dynamically surface plasmon polaritons $s^{6,7}$. Recently, self-accelerating light beams have been extended from the paraxial limit to a nonparaxial regime, which are the solutions of the wave equation under different coordinates ${ }^{8-10}$, and even to arbitrary trajectories which are based on Caustic theory ${ }^{11,12}$.

Notwithstanding the recent progress in optics, accelerating acoustic beams and potential applications in acoustics have not been considered. Actually, accelerating acoustic beam, if realized, will open new design possibilities for acoustic devices and have deep implications in acoustical applications where special control of sound waves is needed. In the scope of medical ultrasonics, for instance, acoustic energy can be delivered to kill cancer cells without affecting healthy tissue in front of the cancer cells. A cylindrically symmetric accelerating beam such as the ring-Airy beam ${ }^{13}$ can be utilized to generate an axisymmetric focused wave without using acoustic gradient index lenses ${ }^{14}$. Although it has been proposed that the fundamental concept of accelerating beams can be applied to linear wave systems ranging from electromagnetic and elastic waves to matter waves ${ }^{9}$, no experimental realizations in acoustics have been published.

In optics, accelerating optical beams such as the Airy beam ${ }^{3}$, non-paraxial accelerating beam ${ }^{8}$ and non-paraxial Mathieu and Webber beams ${ }^{9}$ are experimentally generated by manipulating the phase profile in the spatial Fourier spectrum of the boundary condition. This method cannot be directly applied to acoustics because it is difficult to conduct the Fourier transformation or to manipulate the phase profile of the sound wave in the propagation process. A more intuitive way to generate arbitrary accelerating micron-scale beam by adjusting the spatial phase profile of an incident Gaussian beam with the Spatial Light Modulator (SLM) is proposed and the arbitrary accelerating light beams are experimentally observed in both two and three dimensions ${ }^{12}$. This scheme is also utilized to generate arbitrary bending plasmonic light waves ${ }^{15}$ which use a binary plasmonic phase mask to couple the free space light to the surface plasmon. However, the scheme cannot be directly utilized to generate bending acoustic waves in homogeneous fluid media, which is of interest to researchers in acoustic levitation ${ }^{16}$, medical imaging ${ }^{17}$ as well as therapeutic ultrasound ${ }^{18}$.

In this report, the experimental observation of accelerating acoustic beams along arbitrary convex trajectories is reported. Caustic theory ${ }^{12}$ and geometrical properties are utilized to construct the relationship between an arbitrary convex trajectory and the spatial phase profile of a continuous line acoustics source on the boundary. 


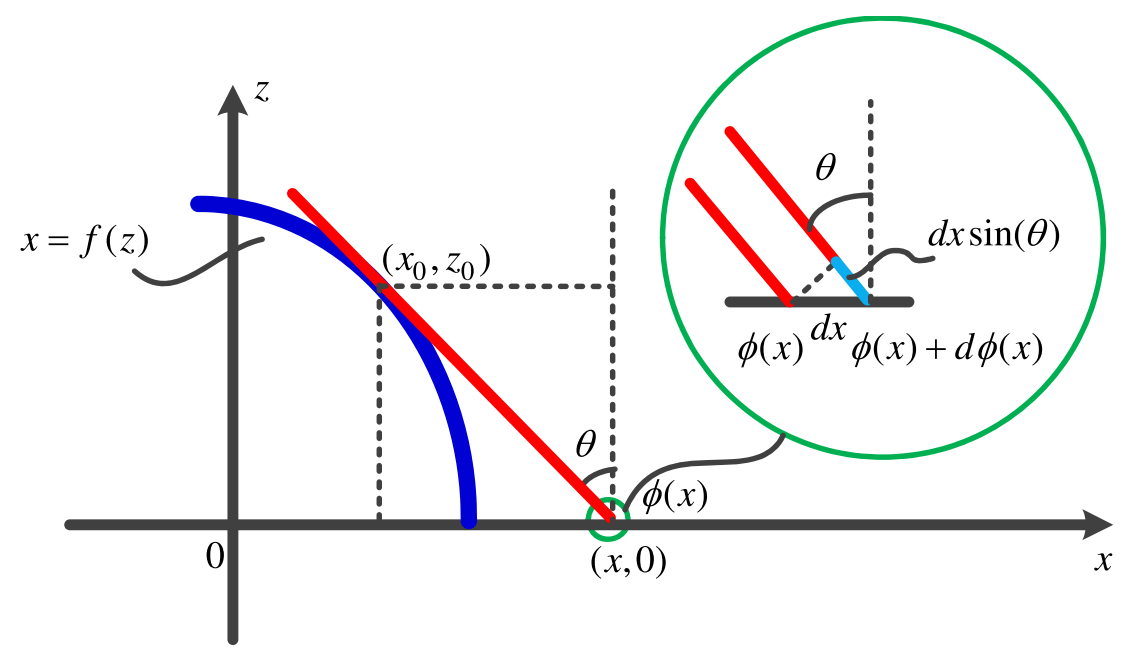

Figure $1 \mid$ Illustration of an arbitrary convex trajectory $x=f(z)$ and the tangent line.

Both paraxial and non-paraxial regimes are investigated and compared with the Airy beam ${ }^{3}$ and non-paraxial accelerating beam $^{8}$, respectively. The sound field distribution is formulated with the Green function and the integral equation method ${ }^{19}$. The continuous source on the boundary is sampled spatially and generated by loudspeakers which are modelled as point sound sources. The simulation and experimental results are in good agreement with the theory. The scattering effect of a rigid sphere in the sound field is also investigated and it is demonstrated that the approach is robust against scattering caused by obstacles.

\section{Results}

Derivation of the spatial phase profile on the boundary. Consider the coordinate system depicted in Fig. $1, x=f(z)$ denotes an arbitrary convex trajectory (blue line), and $\phi(x)$ is the spatial phase profile on the boundary (the $x$ axis). Suppose that $\left(x_{0}, z_{0}\right)$ is a point on the trajectory and $\theta$ is the angle between the $z$-axis and the tangent line (red line) through the point $\left(x_{0}, z_{0}\right)$. The cross point of the tangent line and the $x$-axis is denoted as $(x, 0)$.

The trajectory can be viewed as a caustic which is defined as an envelope to a family of tangents ${ }^{12}$ that relate each point $\left(x_{0}, z_{0}\right)$ on the curve to the point $(x, 0)$ at the plane $z=0$. According to the geometrical property illustrated in Fig. 1, the slope of the tangent line can be expressed as,

$$
f^{\prime}\left(z_{0}\right)=-\tan (\theta),
$$

where $f^{\prime}(z)$ is the first order derivative of $f(z)$. The intercept of the tangent line on the $x$ axis can be obtained as,

$$
x=x_{0}+z_{0} \tan (\theta)
$$

where $x_{0}=f\left(z_{0}\right)$. Combining Equations (1) and (2) to eliminate the variable $z_{0}$, one can readily deduce the functional relationship between the transverse intercept $x$ and the angle $\theta$ as $\tan (\theta)=g(x)$.

The general form of the Fermat's principle states that the derivative of the phase accumulated along the actual wave path will be zero with respect to infinitesimal variations of the path ${ }^{20,21}$. Therefore, the phase relation as depicted inside the green line circle in Fig. 1 can be described by,

$$
\phi(x)+d \phi(x)+k d x \sin (\theta)=\phi(x),
$$

where $d \phi(x), d x$ represent the phase shift and the infinitesimal distance between two cross points along the $x$ direction respectively, and $k$ is the wavenumber.

After simplifying Equation (3), the relation between the spatial phase profile $\phi(x)$ and the angle $\theta$ can be deduced as,

$$
\frac{d \phi(x)}{d x}=-k \sin (\theta) \text {. }
$$

When the paraxial limit (i.e. $\theta<20^{\circ}$ ) is considered, the approximation $\tan (\theta) \sim \sin (\theta)$ is valid as the relative error $[\tan (\theta)-\sin (\theta)] /$ $\sin (\theta)$ is less than $7 \%$ for angles where $\theta<20^{\circ}$. By combining $\tan (\theta)$ $=g(x)$ and Equation (4), the derivative of the spatial phase profile on the boundary could be written as $^{12}$,

$$
\frac{d \phi(x)}{d x}=-k g(x)
$$
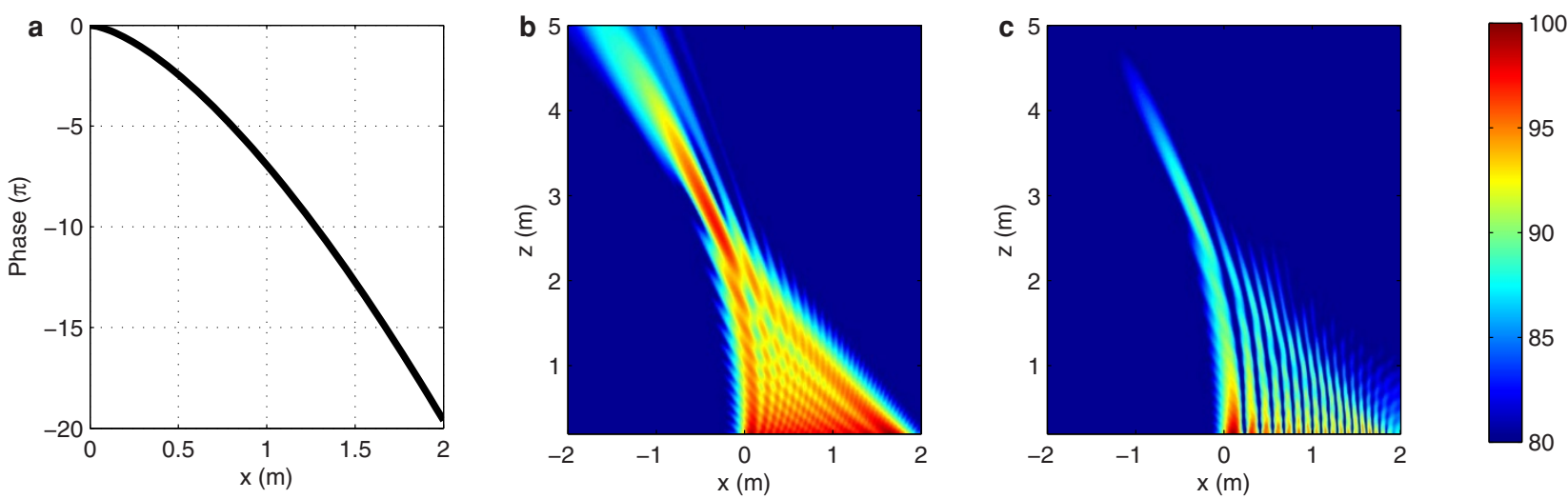

Figure $2 \mid$ Paraxial accelerating acoustic beam $(f=4 \mathrm{kHz})$. (a) Spatial phase profile along $x$ direction for a parabolic trajectory with $a=0.05$. (b) Simulated sound field distribution $(\mathrm{dB})$ for the parabolic trajectory with $a=0.05$. (c) Airy beam (dB) with scale factor $x_{0}=0.1$. 

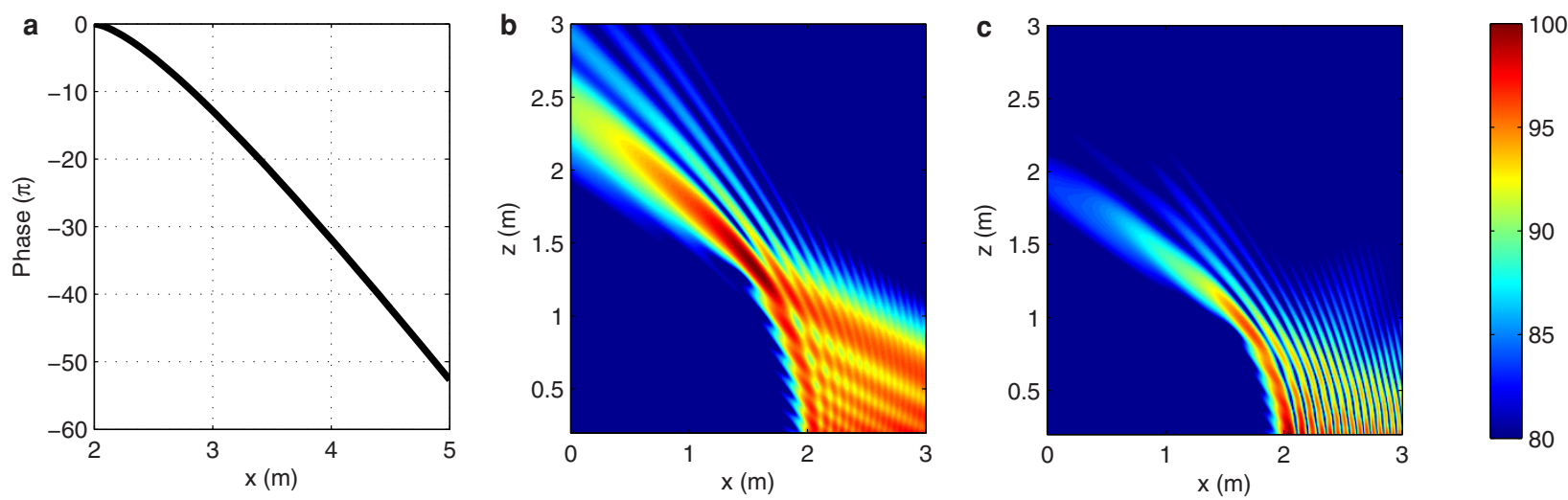

Figure 3 Non-paraxial accelerating acoustic beam $(f=4 \mathrm{kHz})$. (a) Spatial phase profile along the $x$ direction for a circular trajectory with a radius of $2 \mathrm{~m}$. (b) Simulated sound field distribution $(\mathrm{dB})$ for the circular trajectory with a radius of $2 \mathrm{~m}$. (c) Non-paraxial accelerating beam (dB) with order $\beta=100$.

When it comes to the non-paraxial regime, the property of the trigonometric function $\sin (\theta)=\tan (\theta) / \sqrt{1+\tan ^{2}(\theta)}$ can be exploited. With the relation $\tan (\theta)=g(x)$ applied, Equation (5) can be modified as ${ }^{12}$,

$$
\frac{d \phi(x)}{d x}=\frac{-k g(x)}{\sqrt{1+g^{2}(x)}} .
$$

Then the spatial phase profile on the $x$ axis can be obtained by integrating Equations (5) and (6) for paraxial and non-paraxial regimes, respectively. The boundary condition can be determined when the spatial phase profile is deduced, and the acoustic beam along the trajectory $x=f(z)$ can be expected.

Generation of the sound field distribution. To compute the sound field generated by the sound sources with the aforementioned spatial phase profile, the Green function and the integral equation method is utilized $^{19}$,

$$
p(\boldsymbol{r})=\int A e^{i \phi\left(x_{s}\right)} G\left(\boldsymbol{r}, x_{s}, z_{s}=0\right) d x_{s},
$$

where $i$ is the imaginary unit, $A$ is the constant amplitude and $G\left(\boldsymbol{r}, \boldsymbol{r}_{s}\right)$ is the Green function from the sound source located at $r_{s}=\left(x_{s}, z_{s}\right)$ to the field point at $r$. In the free field condition, the Green function is represented as,

$$
G\left(\boldsymbol{r}, \boldsymbol{r}_{s}\right)=\frac{e^{i k\left\|\boldsymbol{r}-\boldsymbol{r}_{s}\right\|}}{4 \pi\left\|\boldsymbol{r}-\boldsymbol{r}_{s}\right\|},
$$

where $\||| \mid$ is the Euclidean norm of the vector.

While there is a rigid sphere in front of the boundary, the scattering effect must be taken into account. The Green function considering the scattering effect is modified to be ${ }^{22}$ (see Supplementary Note 1),

$$
\begin{aligned}
& G_{s}\left(\boldsymbol{r}, \boldsymbol{r}_{s}\right)= \\
& i k \sum_{n=0}^{\infty} \sum_{m=-n}^{n}\left[j_{n}\left(k r_{<}\right)-\frac{j_{n}^{\prime}\left(k r_{0}\right)}{h_{n}^{\prime}\left(k r_{0}\right)} h_{n}\left(k r_{<}\right)\right] h_{n}\left(k r_{>}\right) Y_{n}^{m *}\left(\theta_{s}, \phi_{s}\right) Y_{n}^{m}(\theta, \phi),
\end{aligned}
$$

where $r_{0}$ is the radius of the rigid sphere, $j_{n}()$ is the $n$-th order spherical Bessel function, $h_{n}()$ is the $n$-th order spherical Hankel function of the first kind, $j_{n}^{\prime}()$ and $h_{n}^{\prime}()$ is the first derivative of $j_{n}()$ and $h_{n}()$, respectively, $Y_{n}^{m}()$ are the spherical harmonics, $r_{<}=\min \left(r, r_{s}\right)$ and $r_{>}=\max \left(r, r_{s}\right)$.

When Equations (8) and (9) are substituted into Equation (7), the sound field distribution in the free field condition and with the scattering effect of a rigid sphere considered can be obtained, respectively.
Paraxial and non-paraxial regime. Under the paraxial approximation, the parabolic trajectory is considered. The parabolic curve can be represented as $f(z)=-a z^{2}$ ( $a$ is constant), so one can obtain the spatial phase profile $\phi(x)=-4 / 3 a^{1 / 2} k x^{3 / 2}$ (see Supplementary Note 2). The spatial phase profile is illustrated in Fig. 2 (a). The sound field distribution in free field condition is presented in Fig. 2 (b) when $a=$ 0.05 . Figure 2 (c) shows the result of the Airy Beam ${ }^{1-3}$ which accelerates along a parabolic trajectory. Actually, the Airy function has the asymptotic form expressed as Equation (10) when $x \gg x_{0}{ }^{1}$. The phase obeys the same law with aforementioned spatial phase profile.

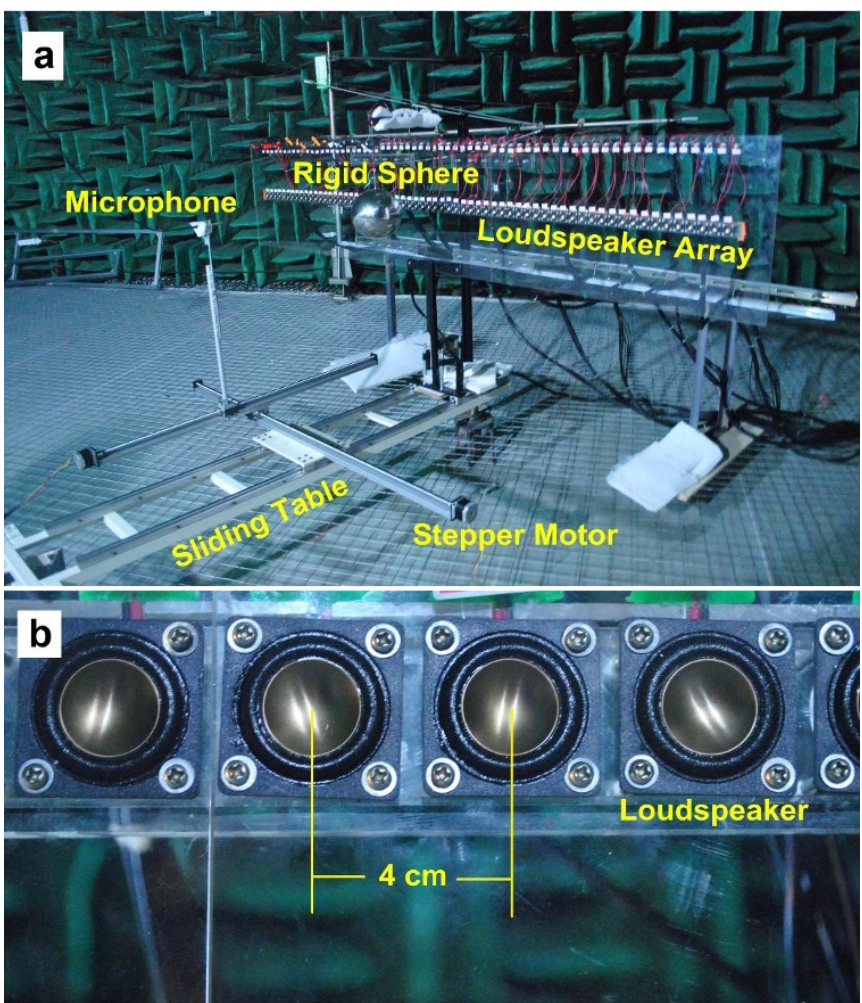

Figure $4 \mid$ Experimental setup. (a) The panorama view of the experimental facility, the rigid sphere is removable for the experiments without obstacle scattering. (b) The loudspeakers are spaced $4 \mathrm{~cm}$ apart.

(Acknowledgements go to Y.H. for taking the photographs.) 

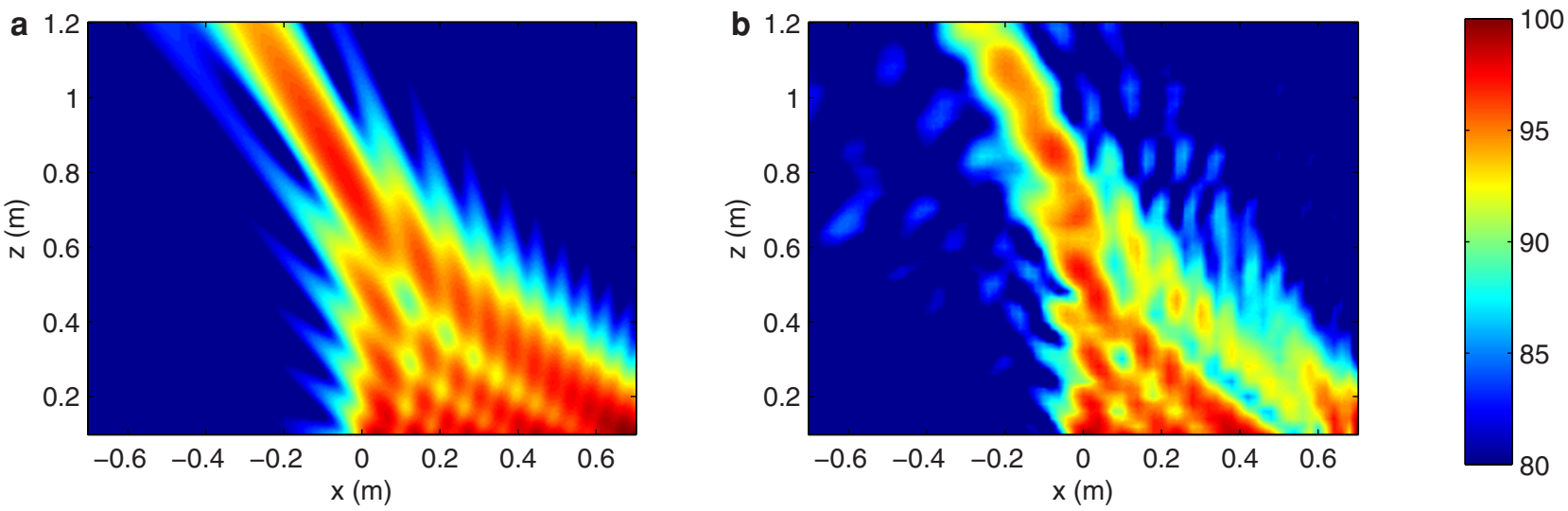

Figure $5 \mid$ Paraxial accelerating acoustic beam $(\mathrm{dB})$ along a parabolic trajectory with $\mathbf{a}=\mathbf{0 . 1 5}(f=4 \mathrm{kHz})$. (a) Simulated sound field distribution. (b) Experimental sound field distribution.

$$
A i\left(-\frac{x}{x_{0}}\right) \approx \frac{1}{\sqrt{\pi}}\left(\frac{x}{x_{0}}\right)^{1 / 4} \sin \left[\frac{2}{3}\left(\frac{x}{x_{0}}\right)^{3 / 2}+\frac{\pi}{4}\right],
$$

where $A i()$ is Airy function and $x_{0}$ is a scale factor.

In the non-paraxial regime, it is more complicated to use Equation (6) instead of Equation (5), but it is still solvable when the trajectory is relatively simple. For example, consider a circle situated at $(0,0)$ with the radius $a$. The circle can be described as,

$$
f(z)=\sqrt{a^{2}-z^{2}} .
$$

By applying Equation (11) to Equations (1)-(5), one can obtain the spatial phase profile $\phi(x)=-k\left[\left(x^{2}-a^{2}\right)^{1 / 2}+a \arcsin (a / x)\right]$ (see Supplementary Note 3 ). The spatial phase profile is depicted in Fig. 3 (a) and the simulated sound field distribution is illustrated in Fig. 3 (b) when $a=2 \mathrm{~m}$. Figure 3 (c) presents the result of Non-paraxial Accelerating Beam (NAB) ${ }^{9,10}$ with the boundary condition,

$$
\left.p(x, z)\right|_{z=0}=J_{\beta}[k(x-a)+\beta],
$$

where $p$ is sound field pressure and $\beta$ is the order of Bessel function.

Experimental validations. Experiments were conducted to verify the simulation results with a 64 channel loudspeaker array. The phases of the excitation signals fed to the loudspeakers are adjusted according to the necessary sampled spatial phase profile on the boundary to generate the corresponding convex trajectory while the amplitudes are uniform. The experimental setup is depicted in Fig. 4.

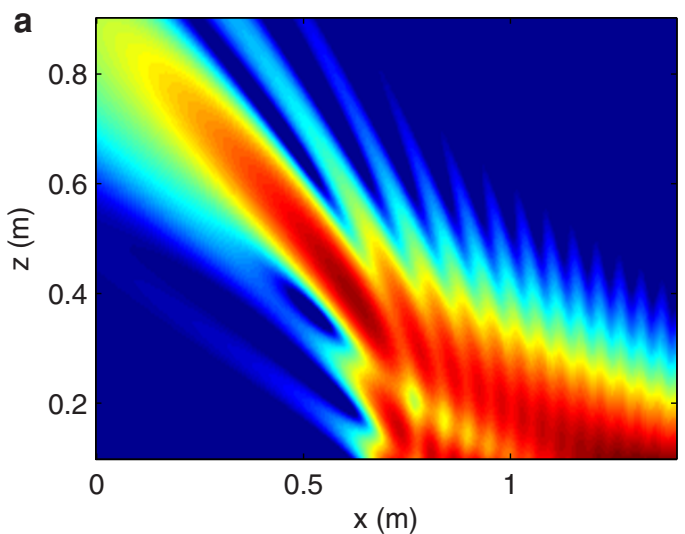

The experimental results of the parabolic trajectory under the paraxial approximation and the circular trajectory for the non-paraxial regime are illustrated in Figs. 5 and 6, respectively. The relative error $e_{1}=\mathrm{E}\left[\left|\mathrm{L}_{\mathrm{ps}}-\mathrm{L}_{\mathrm{pe}}\right|\right] / \mathrm{E}\left[\mathrm{L}_{\mathrm{ps}}\right] \times 100 \%(\mathrm{E}[]$ represents the expectation operator) between the experimental sound pressure level $\mathrm{L}_{\mathrm{pe}}$ and the simulated sound pressure level $\mathrm{L}_{\mathrm{ps}}$ for the parabolic trajectory and the circular trajectory, are $5.1 \%$ and $4.6 \%$, respectively. Considering the unavoidable scattering of the plexiglass panel, the metal support structure and the sliding table, the relative error less than $10 \%$ is rather low, which demonstrates that the experimental results are in good agreement with the simulations.

Scattering of the rigid sphere. For simplicity, the obstacle is supposed to be a rigid sphere and the trajectory is a circle centred at $(0, a)$ with the radius $a$. Such a circle can be represented as,

$$
f(z)= \pm \sqrt{a^{2}-(z-a)^{2}}
$$

By applying Equation (13) to Equations (1)-(5), one can obtain the spatial phase profile $\phi(x)=-k[|x|-2 a \arctan (|x| / a)]$ (see Supplementary Note 4$)$, where || is the absolute value operator. The simulation and experimental results with and without scattering effect are depicted in Fig. 7. Due to the limitation of the measurement range of the experimental equipment, the radius here was set to be $0.32 \mathrm{~m}$. The black dashed circles in Fig. 7 (c) and Fig. 7 (d) represent the location of the rigid sphere with a radius of $0.1 \mathrm{~m}$. Due to the presence of the sphere and the size of the measurement equipment, the sound field distribution is only measured outside the white square with side length $0.17 \mathrm{~m}$.
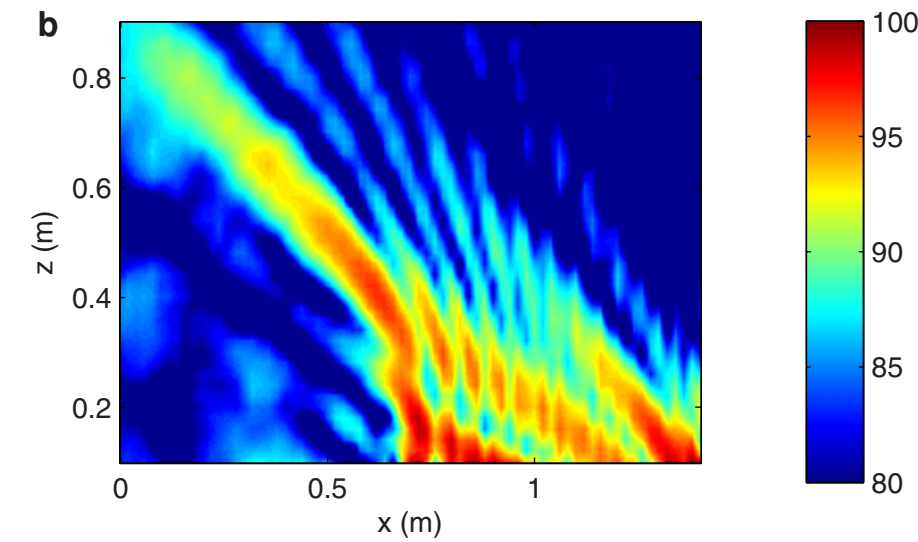

Figure 6 Non-paraxial accelerating acoustic beam (dB) along a circular trajectory with a radius of $0.7 \mathrm{~m}(f=4 \mathrm{kHz})$. (a) Simulated sound field distribution. (b) Experimental sound field distribution. 

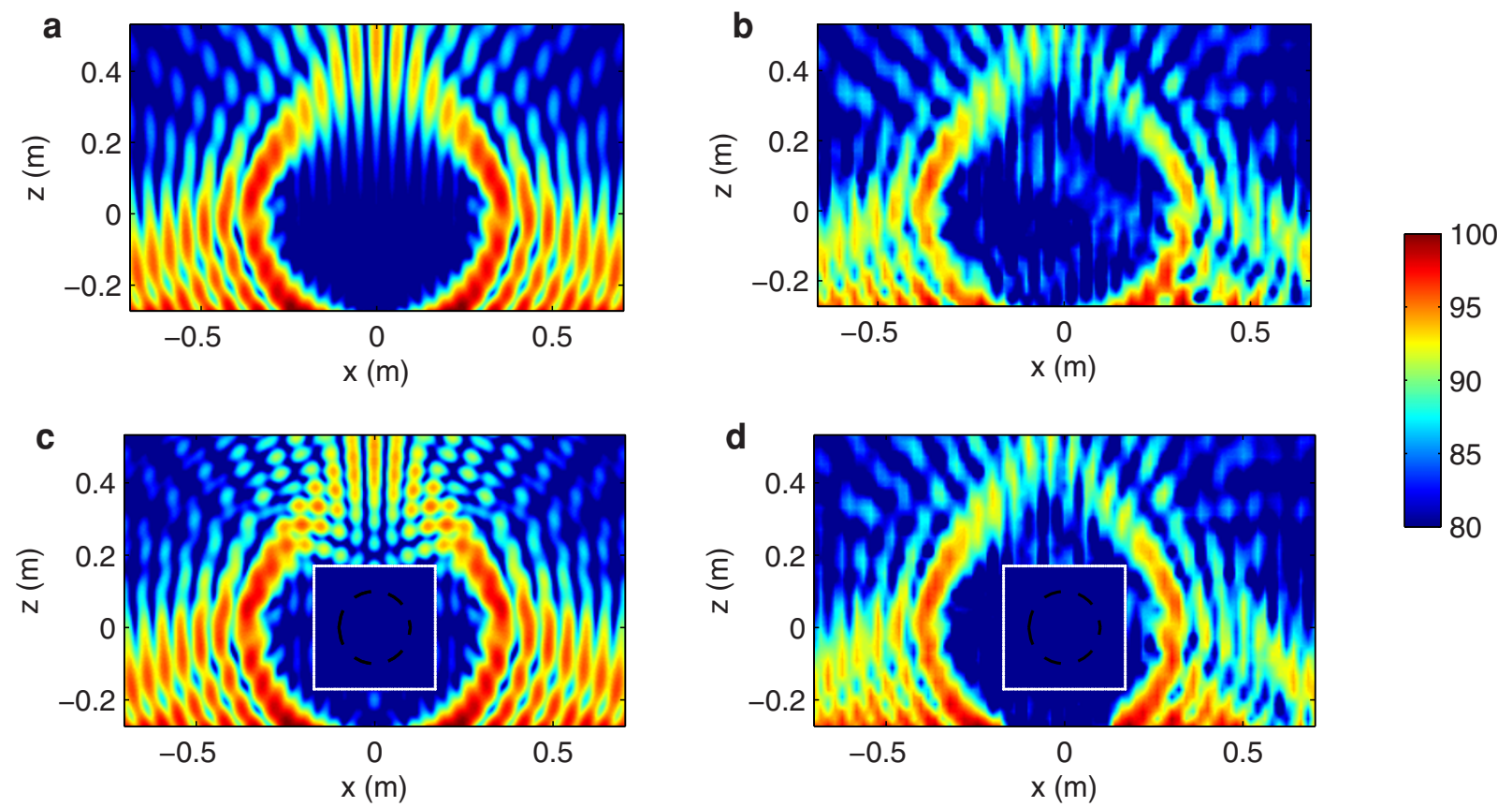

Figure $7 \mid$ Accelerating acoustic beam (dB) along a circular trajectory with a radius of $0.32 \mathrm{~m}(f=4 \mathrm{kHz})$. (a) Simulated sound field distribution without scattering effect. (b) Experimental sound field distribution without scattering effect. (c) Simulated sound field distribution with scattering effect. (d) Experimental sound field distribution with scattering effect. The black dashed circle in (c) and (d) depicts the rigid sphere with radius $0.1 \mathrm{~m}$. The measurement range is outside the white squares in (c) and (d); no data are measured inside the white squares.

The relative error $e_{1}$ between the experimental and simulation results are $6.9 \%$ and $6.1 \%$, with and without the scattering caused by the rigid sphere, respectively, which show the good agreement between the experimental and simulation results. On the other hand, the relative error $e_{2}=\mathrm{E}\left[\left|\mathrm{L}_{\mathrm{pwo}}-\mathrm{L}_{\mathrm{pwi}}\right|\right] / \mathrm{E}\left[\mathrm{L}_{\mathrm{pwo}}\right] \times 100 \%$ (E[] represents the expectation operator) between the sound pressure level with the rigid sphere scattering $L_{p w i}$ and the sound pressure level without the rigid sphere scattering $\mathrm{L}_{\mathrm{pwo}}$ are $2.0 \%$ and $5.0 \%$, for the simulation results and the experimental results, respectively, which manifest that the approach is robust against scattering effect caused by the possible obstacle.

\section{Discussion}

In principle, the approach proposed in this report is valid for a broad band signal as well and the higher the frequency is, the better the result will be. However, when the frequency is higher and the size of the desired trajectory is larger, more loudspeakers will be needed to discretize and sample the spatial phase profile. It has been reported ${ }^{23}$ that in order to avoid spatial aliasing, the distance between the loudspeakers should not be larger than half the wave length. In this report, a frequency of $4 \mathrm{kHz}$ is chosen because the half wave length $0.043 \mathrm{~m}$ is larger than the loudspeaker interval of $0.04 \mathrm{~m}$.

The scheme is easily extended to a 3D situation with planar loudspeaker arrays and volume caustics ${ }^{12}$. Then the ring-Accelerating beam like the ring-Airy beam ${ }^{13}$ can be generated and this can be used to produce axisymmetric focused wave without using acoustic gradient index lenses ${ }^{14}$. The proposed method utilizes boundaries instead of an entire propagation region to manipulate the wavefront, so the complexity in wavefront engineering is reduced significantly and the application of wave manipulation is expanded.

In conclusion, an intuitive method to generate accelerating acoustic beams by manipulating the spatial phase profile on the boundary has been investigated. The method is based on the Caustic theory ${ }^{12}$ and geometrical properties. The convex trajectories are projected to the spatial phase profile on the boundary which are discretized and sampled spatially. The discretized phase is generated by a loudspeaker array and the excitation signals of the loudspeakers are sine waves whose initial phases are manipulated according to the discretized spatial phase profile to generate the sound field along the convex trajectory. The relative error between the experimental and simulation results is less than $7 \%$ for the parabolic and circular trajectories, which demonstrates good agreement between the experimental and simulation results. The relative error between the sound field with and without the rigid sphere scattering is $5 \%$ for the experimental results, which show good robustness against the scattering effect caused by obstacles.

\section{Methods}

Experimental Setup. The experiments were conducted in an anechoic room. In the experiments, the excitation signals were generated on a PC and transmitted to the loudspeakers through PS PXI 3381 multichannel analogue output cards and power amplifiers. The loudspeakers are mounted on a plexiglass panel for stability and the sound field distribution is measured by a free field microphone carried by a stepper motor which is controlled by the PC. 26 and 32 loudspeakers were used to generate the paraxial parabolic trajectory (Fig. 5) and the non-paraxial circular trajectory (Fig. 6), respectively. For the experimental validation of the robustness to obstacle scattering (Fig. 7), 64 loudspeakers were utilized. The loudspeakers were spaced at an interval $0.04 \mathrm{~m}$ and the frequency used for all the above-mentioned experiments was $4 \mathrm{kHz}$.

1. Berry, M. V. \& Balazs, N. L. Nonspreading wave packets. Am. J. Phys. 47, 264 (1979).

2. Siviloglou, G. A. \& Christodoulides, D. N. Accelerating finite energy Airy beams. Opt. Lett. 32, 979 (2007).

3. Siviloglou, G. A., Broky, J., Dogariu, A. \& Christodoulides, D. N. Observation of accelerating Airy beams. Phys. Rev. Lett. 99, 213901 (2007).

4. Baumgartl, J., Mazilu, M. \& Dholakia, K. Optically mediated particle clearing using Airy wavepackets. Nature Photon. 2, 675 (2008).

5. Polynkin, P., Kolesik, M., Moloney, J. V., Siviloglou, G. A. \& Christodoulides, D. N. Curved plasma channel generation using ultraintense Airy beams. Science 324, 229 (2009).

6. Minovich, A. et al. Generation and near-field imaging of Airy surface plasmons. Phys. Rev. Lett. 107, 116802 (2011).

7. Li, L., Li, T., Wang, S. M., Zhang, C. \& Zhu, S. N. Plasmonic Airy beam generated by In-plane diffraction. Phys. Rev. Lett. 107, 126804 (2011).

8. Kaminer, I., Bekenstein, R., Nemirovsky, J. \& Segev, M. Nondiffracting accelerating wave packets of Maxwell's Equations. Phys. Rev. Lett. 108, 163901 (2012). 
9. Zhang, P. et al. Nonparaxial Mathieu and Webber accelerating beams. Phys. Rev. Lett. 109, 193901 (2012).

10. Zhang, P. et al. Generation of linear and nonlinear nonparaxial accelerating beams. Opt. Lett. 37, 14 (2012).

11. Greenfield, E., Segev, M., Walasik, W. \& Raz, O. Accelerating light beam along arbitrary convex trajectories. Phys. Rev. Lett. 106, 213902 (2011).

12. Froehly, L. et al. Arbitrary accelerating micron-scale caustic beams in two and three dimensions. J. M. Opt. Exp. 19, 16455 (2011).

13. Panagiotopoulos, P., Papazoglou, D. G., Couairon, A. \& Tzortzakis, S. Sharply autofocused ring-Airy beams transforming into non-linear intense light bullets. Nature Commun. 4, 2622 (2013).

14. Romero-Garcia, V. et al. Wave focusing using symmetry matching in axisymmetric acoustic gradient index lenses. Appl. Phys. Lett. 103, 264106 (2013).

15. Epstein, I. \& Arie, A. Arbitrary bending plasmonic light waves. Phys. Rev. Lett. 112, 023903 (2014).

16. Brandt, E. H. Levitation in physics. Science 243, 349-355 (1989).

17. Lu, J. Y. \& Greenleaf, J. F. Ultrasonic nondiffracting transducer for medical imaging. IEEE Trans. Ultrason. Ferroelectre. Freq. Control 37, 438-447 (1990).

18. Speed, C. A. Therapeutic ultrasound in soft tissue lesions. Rheumatology 40, 1331-1336 (2001).

19. Morse, P. M. \& Ingard, K. U. Theoretical Acosutics. (McGraw-Hill Inc., New York, 1968).

20. Yu, N. et al. Light propagation with phase discontinuities: generalized laws of reflection and refraction. Science 334, 333 (2011).

21. Li, Y., Liang, B., Gu, Z., Zou, X. \& Cheng, J. Reflected wavefront manipulation based on ultrathin planar acoustic metasurfaces. Sci. Rep. 3, 2546; doi:10.1038/ srep02546 (2013).

22. Bowman, J. J., Senior, T. B. A. \& Uslenghi, P. L. E. Electromagnetic and Acoustic Scattering by Simple Shapes. (John Wiley, New York, 1969).
23. Nelson, P. A. \& Elliott, S. J. Active Control of Sound. (Academic Press, Massachusetts, 1993).

\section{Acknowledgments}

This work was supported by the National Basic Research Program of China (973 Program) (Grant No. 2010CB327803), National Natural Science Foundation of China (Grant Nos. $11174138,11374157,11374156)$.

\section{Author contributions}

J.C. initiated the research. S.Z. and X.Q. conducted the theoretical analysis. Y.H. and J.L. designed and carried out the experiments. S.Z., J.L., X.Q. and J.C. wrote the manuscript. J.L., X.Q. and J.C. supervised the study. I.B. revised the manuscript and commented on the paper.

\section{Additional information}

Supplementary information accompanies this paper at http://www.nature.com/ scientificreports

Competing financial interests: The authors declare no competing financial interests.

How to cite this article: Zhao, S. et al. Delivering Sound Energy along an Arbitrary Convex Trajectory. Sci. Rep. 4, 6628; DOI:10.1038/srep06628 (2014).

This work is licensed under a Creative Commons Attribution-NonCommercialNoDerivs 4.0 International License. The images or other third party material in this article are included in the article's Creative Commons license, unless indicated otherwise in the credit line; if the material is not included under the Creative Commons license, users will need to obtain permission from the license holder in order to reproduce the material. To view a copy of this license, visit http:// creativecommons.org/licenses/by-nc-nd/4.0/ 